\title{
Inhibitory effects of Picrasma quassioides (D.Don) Benn. on airway inflammation in a murine model of allergic asthma
}

\author{
NA-RAE SHIN ${ }^{1,2}$, IN-SIK SHIN ${ }^{1}$, CHAN-MI JEON ${ }^{1,3}$, JU-MI HONG $^{1,4}$, \\ SEI-RYANG OH ${ }^{1}$, KYU-WOUNG HAHN ${ }^{2}$ and KYUNG-SEOP AHN ${ }^{1}$ \\ ${ }^{1}$ Natural Medicine Research Center, Korea Research Institute of Bioscience and Biotechnology, Cheongwon-gun, \\ Chungbuk 363-883; ${ }^{2}$ Department of Biological Sciences and Biotechnology, College of Life Science and \\ Nano Technology, Hannam University, Daejeon 306-791; ${ }^{3}$ Department of Pharmacy, College of Pharmacy, \\ Chungnam National University, Daejeon 305-764; ${ }^{4}$ Department of Pharmacy, College of Pharmacy, \\ Chungbuk National University, Cheongju, Chungbuk 361-763, Republic of Korea
}

Received September 3, 2013; Accepted March 12, 2014

DOI: $10.3892 / \mathrm{mmr} .2014 .2322$

\begin{abstract}
Picrasma quassioides (D.Don) Benn. (PQ) is used in traditional medicine for the treatment of inflammatory conditions, including gastritis. This study aimed to evaluate the inhibitory effects of PQ on the inflammatory responses in mice with allergic asthma induced by ovalbumin (OVA) and in lipopolysaccharide (LPS)-stimulated RAW264.7 cells. To induce allergic asthma, the mice underwent OVA sensitization on days 0 and 14 and then were challenged with OVA from days $21-23$. The mice were administered 15 and $30 \mathrm{mg} / \mathrm{kg}$ doses of PQ $1 \mathrm{~h}$ prior to the OVA challenge. The PQ treatment decreased the inflammatory cell count in the bronchoalveolar lavage fluid of the mice and reduced the levels of interleukin (IL)-4, IL-5, IL-13 and immunoglobulin (Ig)E when compared with those in the OVA group. The PQ treatment also reduced the airway hyperresponsiveness induced by the OVA challenge, attenuated the recruitment of inflammatory cells and the mucus production in the airways of the mice. In the LPS-stimulated RAW264.7 cells, the PQ treatment reduced the overexpression of inducible nitric oxide synthase (iNOS). The results indicated that PQ inhibits inflammatory responses in mice with OVA-sensitized/challenged allergic asthma and in LPS-stimulated RAW264.7 cells. These effects were consid-
\end{abstract}

Correspondence to: Professor Kyu-Woung Hahn, Department of Biological Sciences and Biotechnology, College of Life Science and Nano Technology, Hannam University, 70 Hannam-ro, Daejeon 306-791, Republic of Korea

E-mail: hahnkw@hnu.kr

Dr Kyung-Seob Ahn, Natural Medicine Research Center, Korea Research Institute of Bioscience and Biotechnology, 30 Yeongudanji-ro, Cheongwon-gun, Chungbuk 363-883, Republic of Korea

E-mail: ksahn@kribb.re.kr

Key words: Picrasma quassioides, allergic asthma, airway inflammation, inducible nitric oxide synthase ered to be associated with the suppression of iNOS expression. Therefore, PQ may have the potential to treat airway inflammatory diseases, including allergic asthma.

\section{Introduction}

Inflammation is a complex process involving various mediators. In particular, nitric oxide (NO) is considered as an important inflammatory mediator. NO derived from inducible NO synthase (iNOS) is crucial in airway inflammation (1). Allergic asthma features airway inflammation, airway hyperresponsiveness (AHR) and mucus hypersecretion. The development of allergic asthma results in iNOS overexpression in the airway, which induces asthmatic responses. A previous study demonstrated that iNOS expression indirectly induced the activation of T helper (Th)2 lymphocytes to contribute to the pathophysiological alterations of asthma. The Th2 lymphocytes produced various Th2 cytokines, including interleukin (IL)-4, IL-5 and IL-13. Th2 cytokines were closely associated with the development of allergic asthma via the secretion of allergen-specific immunoglobulin $(\mathrm{Ig}) \mathrm{E}$, chemokines, proinflammatory mediators and chemoattractants (2).

Picrasma quassioides (D.Don) Benn. (PQ) is a medicinal herb belonging to the family Simaroubaceae. In China, PQ is used as a traditional herbal medicine for the treatment of numerous diseases, including diarrhea, dysentery, inflammation, microbial infection and fever. Previous studies have shown that PQ has anti-inflammatory, anticancer and anti-oxidant effects in in vitro and in vivo experiments $(3,4)$. Liu et al (5) demonstrated that PQ attenuated inflammatory bowel disease. Additionally, Fan et al (6) showed that PQ inhibited the production of inflammatory mediators in lipopolysaccharide (LPS)-stimulated macrophage cells and an adjuvant-induced model of arthritis. However, to the best of our knowledge, no study has investigated the protective effects of PQ on airway inflammation in an ovalbumin (OVA)-sensitized/challenged allergic asthma model.

Therefore, the present study investigated the effect of PQ on airway inflammation using an OVA-sensitized/challenged allergic asthma murine model by measuring inflammatory cell 
counts, Th2 cytokine and IgE levels, and by performing an histological analysis of the lung tissue of the mice. To investigate the anti-inflammatory mechanism of $\mathrm{PQ}$, the levels of NO, proinflammatory cytokines and iNOS expression were evaluated in the RAW264.7 murine macrophage cell line.

\section{Materials and methods}

Cell culture and cell viability. The RAW264.7 (American Type Culture Collection, Manassas, VA, USA) murine macrophage cell line was maintained in Dulbecco's modified Eagle's medium (DMEM) supplemented with 10\% heat-inactivated fetal bovine serum and antibiotic-antimycotic (Gibco-BRL, Carlsbad, CA, USA), including penicillin, streptomycin and Fungizone at $37^{\circ} \mathrm{C}$ in a $5 \% \mathrm{CO}_{2}$ and $95 \%$ air incubator. The cell viability following PQ exposure was measured using a 3-(4,5-dimethylthiazol-2-yl)-2,5-diphenyltetrazolium bromide (MTT; Amresco, Solon, OH, USA) assay. The RAW264.7 cells were cultured in 96-well plates at a density of $1 \times 10^{4}$ cells/well. The PQ extract was obtained from the Plant Extract Bank at Korea Research Institute of Bioscience and Biotechnology (KRIBB; PB3612.2; Ochang-eup, Korea). The PQ extract was added to each individual well at a concentration of 20,40, 80 or $100 \mu \mathrm{g} / \mathrm{ml}$, and then the plates were incubated for $24 \mathrm{~h}$. MTT solution $(10 \mu \mathrm{l})$ was added to each well, and the plates were incubated for $4 \mathrm{~h}$ at $37^{\circ} \mathrm{C}$. Following incubation, $100 \mu \mathrm{l}$ dimethylsulfoxide was added to each well for the solubilization of formazan. The optical density was measured at $570 \mathrm{~nm}$ using a microplate reader (Molecular Devices Co., Sunnyvale, CA, USA).

Determination of NO production. Cells $\left(5 \times 10^{5}\right.$ cells $\left./ \mathrm{ml}\right)$ were seeded in 96-well plates with phenol red-free DMEM, treated with different concentrations of PQ $(20,40,80$ and $100 \mu \mathrm{g} / \mathrm{ml})$ $1 \mathrm{~h}$ prior to LPS treatment and then incubated in the presence of LPS $(0.5 \mu \mathrm{g} / \mathrm{ml})$ for $24 \mathrm{~h}$. The nitrite accumulation in the culture medium was measured using the Griess Reagent system (Promega Corporation, Madison, WI, USA). The absorbance at $540 \mathrm{~nm}$ was measured using a microplate reader (Molecular Devices, LLC).

Immunoblot analysis. The cells were treated as described in the previous sections and then incubated in the presence of LPS $(0.5 \mu \mathrm{g} / \mathrm{ml})$ for 1 or $24 \mathrm{~h}$. The cells were collected by centrifugation, washed twice with phosphate-buffered saline (PBS), and suspended using CelLytic MT Mammalian Tissue Lysis/Extraction Reagent (Sigma-Aldrich, St. Louis, MO, USA) containing protease inhibitors. The protein concentration was determined using a protein assay reagent (Bio-Rad Laboratories, Inc., Hercules, CA, USA), according to the manufacturer's instructions. Equal quantities of total cellular protein $(30 \mathrm{~g})$ were resolved by $10 \%$ sodium dodecyl sulfate-polyacrylamide gel electrophoresis and transferred to nitrocellulose membranes. The membranes were incubated with blocking solution (5\% skimmed milk), followed by an overnight incubation at $4^{\circ} \mathrm{C}$ with the appropriate primary antibody. The following primary antibodies and dilutions were used: Polyclonal anti- $\beta$-actin (anti-rabbit, 1:2,000 dilution; Cell Signaling Inc., Danvers, MA, USA) and polyclonal anti-iNOS (anti-rabbit, 1:200 dilution; Enzo Life Sciences
Inc., Farmingdale, MA, USA). The blots were washed three times with Tris-buffered saline containing Tween-20 (TBST) and then incubated with a 1:10,000 dilution of horseradish peroxidase-conjugated secondary antibody (Jackson ImmunoResearch Laboratories, Inc., West Grove, PA, USA) for $30 \mathrm{~min}$ at room temperature. The blots were washed three times with TBST, and then developed using an enhanced chemiluminescence kit (Thermo Fisher Scientific Inc., San Jose, CA, USA).

Experimental procedure. Specific pathogen-free female BALB/c mice (weight, 19-21 g; age, 6-8 weeks) were purchased from Koatech Co. (Pyeongtaek, Korea). The mice were divided into five groups ( $\mathrm{n}=7$ per group) and housed under standard conditions (temperature, $22 \pm 2^{\circ} \mathrm{C}$; humidity, $55 \pm 5 \%$; 12 -h light/dark cycle) with food and water ad libitum. All experimental procedures were approved by the Institutional Animal Care and Use Committee of KRIBB. The mice were divided into five groups $(n=7)$, and allergic asthma was induced by OVA in four groups. Each mouse was immunized by intraperitoneal injection of $20 \mu \mathrm{g}$ OVA emulsified with $2 \mathrm{mg}$ aluminum hydroxide in $200 \mu$ PBS buffer (pH 7.4) on days 0 and 14. On days $21-23$, the mice were forced to inhale a $1 \%(\mathrm{w} / \mathrm{v})$ OVA solution aerosolized using an ultrasonic nebulizer (NE-U12; Omron Corp., Tokyo, Japan) for $1 \mathrm{~h}$. PQ was administered by oral gavage at dose levels of 15 and $30 \mathrm{mg} / \mathrm{kg} 1 \mathrm{~h}$ prior to the OVA challenge. Dexamethasone was used as the positive control drug, and was administered by oral gavage at a dose of $3 \mathrm{mg} / \mathrm{kg}$.

Measurement of AHR. Twenty-four hours after the final OVA challenge, the AHR was measured by the indirect usage of single-chamber, whole-body plethysmography (Allmedicus Co., Ltd., Seoul, Korea). Each mouse was placed in a plastic chamber and exposed to methacholine aerosols at increasing concentrations (12.5-50 mg/ml in PBS) for $3 \mathrm{~min}$. Following each methacholine challenge, the Penh values were measured for $3 \mathrm{~min}$.

Measurement of inflammatory cell counts in bronchoalveolar lavage (BAL) fluid. To obtain BAL fluid, mice were sacrificed $48 \mathrm{~h}$ after the final OVA challenge with an intraperitoneal injection of phenobarbital $(50 \mathrm{mg} / \mathrm{kg}$; Hanlim Pharm., Co., Seoul, Republic of Korea) and tracheotomy was performed. The trachea was cannulated and the left bronchi were tied for histopathological examination. Following instilling ice-cold PBS $(0.4 \mathrm{ml})$ into the lung, BALF was obtained by three successive aspirations (total volume $1.2 \mathrm{ml}$ ) via tracheal cannulation. The total numbers of inflammatory cells were determined by counting cells in at least five squares of a hemocytometer (Reichert, Buffalo, NY, USA) following the exclusion of dead cells by Trypan blue staining. The differential cell count of the BAL fluid was performed using Diff-Quik ${ }^{\circledR}$ staining reagent (B4132-1A; IMEB Inc., San Marcos, CA, USA), according to the manufacturer's instructions. Blood was collected from the inferior vena cava and centrifuged $\left(200 \mathrm{~g}, 10 \mathrm{~min}, 4^{\circ} \mathrm{C}\right)$. The supernatant was stored at $-70^{\circ} \mathrm{C}$.

Measurement of the levels of cytokines in BAL fluid and IgE in serum. The levels of certain cytokines (IL-4, IL-5 and IL-13) 
in the BAL fluid were measured using ELISA kits (R\&D Systems, Minneapolis, MN, USA), according to the manufacturer's instructions. The levels of total IgE and OVA-specific $\mathrm{IgE}$ in the serum were measured by ELISAs. Microtiter plates were coated with anti-IgE antibodies (anti-mouse IgE; $10 \mathrm{~g} / \mathrm{ml}$; AbD Serotec, Oxford, UK) or OVA (Sigma-Aldrich) in PBS-Tween-20, and incubated with the serum samples. The plates were then washed four times, and $200 \mu \mathrm{l}$ o-phenylenediamine dihydrochloride (Sigma-Aldrich) was added to each well. The plates were incubated for $10 \mathrm{~min}$ in the dark and the absorbance was measured at $450 \mathrm{~nm}$ using a microplate reader (Molecular Devices Co.).

Histopathological analysis of the lung tissue. The left lung tissue was fixed in $4 \%$ (v/v) paraformaldehyde, embedded in paraffin, sectioned at a thickness of $4 \mu \mathrm{m}$, and stained with hematoxylin and eosin solution (Sigma-Aldrich) or periodic acid-Schiff (PAS) (IMEB Inc.) to estimate the levels of inflammation and mucus production, respectively.

Statistical analysis. Data are expressed as the mean \pm standard deviation. The statistical significance was determined using analysis of variance followed by a multiple comparison test with Dunnet's adjustment. $\mathrm{P}<0.05$ was considered to indicate a statistically significant difference.

\section{Results}

$P Q$ reduces the $A H R$ induced by OVA challenge. The OVA-sensitized/challenged mice exhibited a significant increase in AHR with the exposure to increasing concentrations of methacholine compared with that of the normal controls. The dexamethasone-treated mice showed a significant reduction in the AHR compared with that of the OVA-sensitized/challenged mice. The PQ-treated mice also exhibited a reduced AHR at the 25 and $50 \mathrm{mg} / \mathrm{ml}$ methacholine concentrations compared with that of the OVA-sensitized/challenged mice. These reductions were similar to the results of the dexamethasone-treated mice (Fig. 1).

$P Q$ reduces the number of inflammatory cells in BAL fluid. The OVA-sensitized/challenged mice exhibited a significant increase in the number of inflammatory cells, in particular eosinophils, in the BAL fluid compared with that of the normal controls. However, the PQ-treated mice showed a markedly reduced number of inflammatory cells, including eosinophils, macrophages, lymphocytes and neutrophils, in the BAL fluid compared with that of the OVA-sensitized/challenged mice (Fig. 2).

$P Q$ reduces the levels of proinflammatory cytokines in BAL fluid and IgE in serum. The levels of IL-4, IL-5 and IL-13 in the BAL fluid were significantly increased in the OVA-sensitized/challenged mice compared with those in the normal controls. By contrast, the administration of PQ treatment induced a significant reduction in these levels in the BAL fluid compared with those of the OVA sensitized/challenged mice (Fig. 3A). In addition, the levels of total $\operatorname{IgE}$ and OVA-specific $\operatorname{IgE}$ in the serum of the

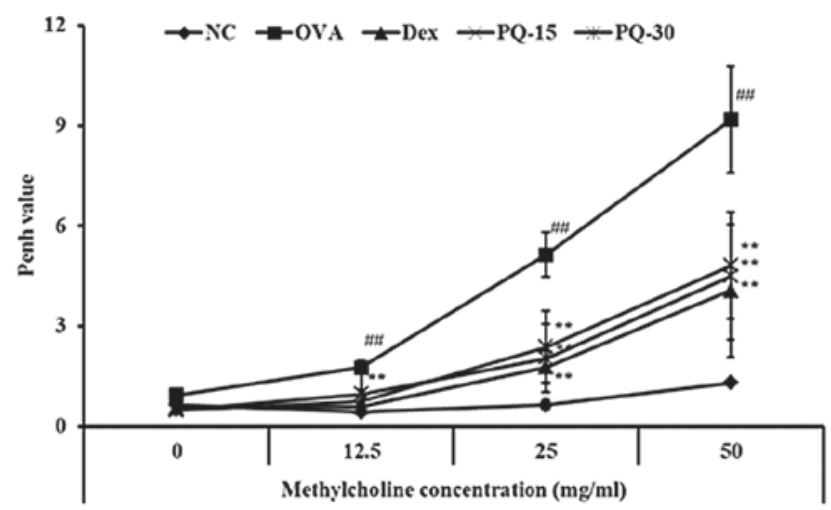

Figure 1. PQ reduced AHR in OVA-sensitized/challenged mice. AHR was measured $24 \mathrm{~h}$ after the lastchallenge in mice administered with various concentrations of methacholine $(12.5-50 \mathrm{mg} / \mathrm{ml})$ using single-chamber, whole-body plethysmography. NC, normal control mice; OVA, OVA-sensitized/challenged mice; Dex, dexamethasone $(3 \mathrm{mg} / \mathrm{kg})+$ OVA-sensitized/challenged mice; PQ-15, PQ (15 mg/kg) + OVA-sensitized/challenged mice; PQ-30, PQ (30 mg/kg) + OVA-sensitized/challenged mice; OVA, ovalbumin; PQ, Picrasma quassioides; AHR, airway hyperresponsiveness. Values are expressed as the mean \pm standard deviation ( $\mathrm{n}=7$ per group). ${ }^{\# \#} \mathrm{P}<0.01$, compared with $\mathrm{NC}$ and ${ }^{* *} \mathrm{P}<0.01$, compared with OVA.

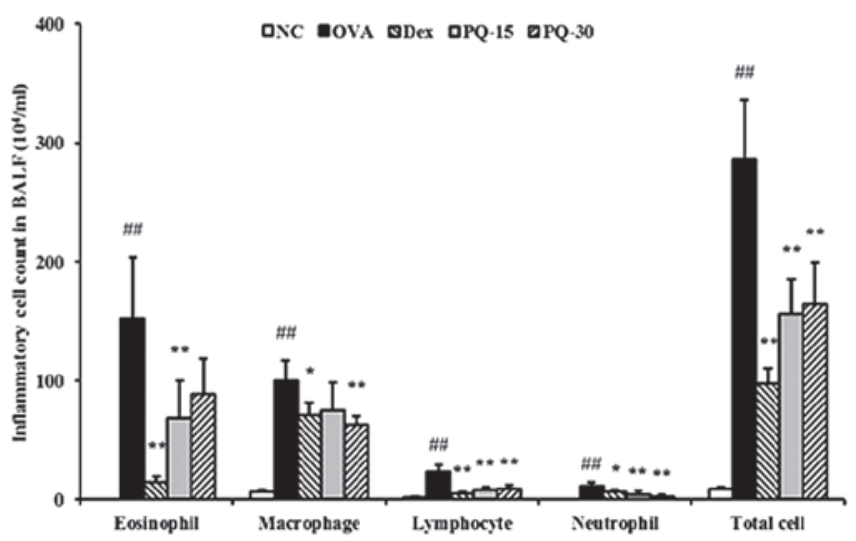

Figure 2. PQ inhibited inflammatory cells in the BAL fluid of the mice. Cells were isolated by centrifugation and stained with Diff-Quik staining reagent. NC, normal control mice; OVA, OVA-sensitized/challenged mice; Dex, dexamethasone (3 mg/kg) + OVA-sensitized/challenged mice; PQ-15, PQ (15 mg/kg) + OVA-sensitized/challenged mice; PQ-30, PQ (30 mg/kg) + OVA-sensitized/challenged mice; BAL, bronchoalveolar lavage; OVA, ovalbumin; PQ, Picrasma quassioides. Values are expressed as the mean \pm standard deviation ( $\mathrm{n}=7 /$ group). ${ }^{\# \#} \mathrm{P}<0.01$, compared with $\mathrm{NC}$; ${ }^{*} \mathrm{P}<0.05$ and ${ }^{* *} \mathrm{P}<0.01$, compared with OVA.

OVA-sensitized/challenged mice were significantly elevated compared with those in the normal controls. However, the PQ-treated mice exhibited a significant reduction in the levels of total IgE and OVA-specific IgE in the serum compared with those in the OVA-sensitized/challenged mice (Fig. 3B and $\mathrm{C}$ ).

$P Q$ decreases the levels of inflammatory cell infiltration and mucus production. Lung tissue from the OVA-sensitized/challenged mice exhibited infiltration of inflammatory cells into peribronchial and perivascular lesions, and mucus hypersecretion from the airway epithelial cells (Fig. 4). By contrast, the lung tissue from the PQ-treated mice showed markedly 

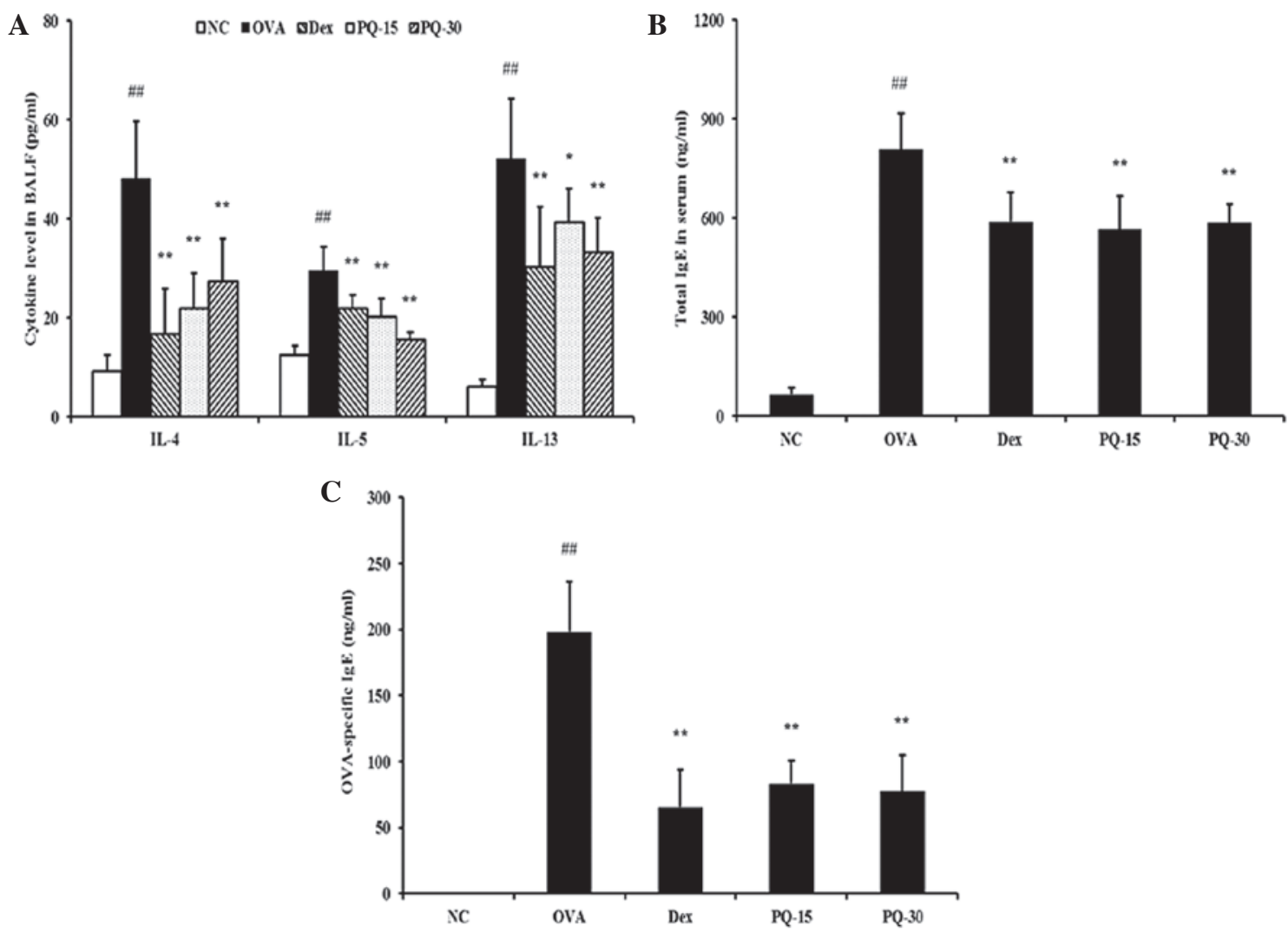

Figure 3. PQ extract inhibited proinflammatory cytokine and IgE levels. (A) Levels of pro-inflammatory cytokines in the BAL fluid, (B) total IgE levels in the serum, (C) OVA-specific IgE levels in the serum determined by ELISA. NC, normal control mice; OVA, OVA-sensitized/challenged mice; Dex, dexamethasone (3 mg/kg) + OVA-sensitized/challenged mice; PQ-15, PQ (15 mg/kg) + OVA-sensitized/challenged mice; PQ-30, PQ (30 mg/kg) + OVA-sensitized/challenged mice; BAL, bronchoalveolar lavage; OVA, ovalbumin; PQ, Picrasma quassioides; Ig, immunoglobulin. Values are expressed as the mean \pm standard deviation (n=7/group). ${ }^{\# /} \mathrm{P}<0.01$, compared with $\mathrm{NC} ;{ }^{*} \mathrm{P}<0.05$ and ${ }^{* *} \mathrm{P}<0.01$, compared with OVA.
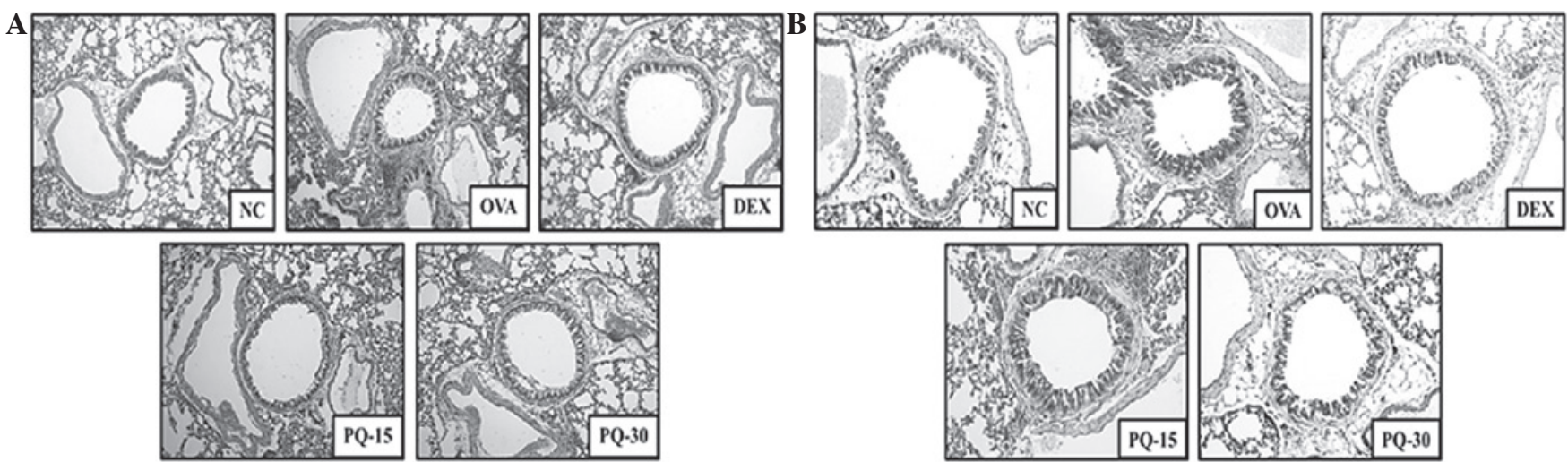

Figure 4. PQ inhibited airway inflammation and mucus production. (A) Histological examination of airway inflammation in the lung tissue by H\&E staining (magnification, x200). (B) Histological examination of mucus production in the lung tissue by PAS staining (magnification, x200). NC, normal control mice; OVA, OVA-sensitized/challenged mice; Dex, dexamethasone (3 mg/kg) + OVA-sensitized/challenged mice; PQ-15, PQ (15 mg/kg) + OVA-sensitized/challenged mice; PQ-30, PQ (30 mg/kg) + OVA-sensitized/challenged mice; OVA, ovalbumin; PQ, Picrasma quassioides; H\&E, hematoxylin and eosin; PAS, periodic acid-Schiff.

lower levels of inflammatory cell infiltration and mucus secretion compared with that from the OVA-sensitized/challenged mice.

$P Q$ inhibits the production of $N O$ in LPS-stimulated RAW264.7 cells. In this study, nontoxic concentrations of PQ (20, 40, 80 and $100 \mu \mathrm{g} / \mathrm{ml}$; Fig. 5A) were tested for their ability to inhibit LPS-stimulated NO production. The
LPS-stimulated cells exhibited an increase in the cellular NO levels compared with those in the non-stimulated cells. However, the cells pretreated with PQ showed inhibition of the production of $\mathrm{NO}$ in a concentration-dependent manner compared with that in the LPS-stimulated cells (Fig. 5B).

$P Q$ suppresses iNOS expression in LPS-stimulated RAW264.7 cells. As shown in Fig. 6, the levels of iNOS protein expression 

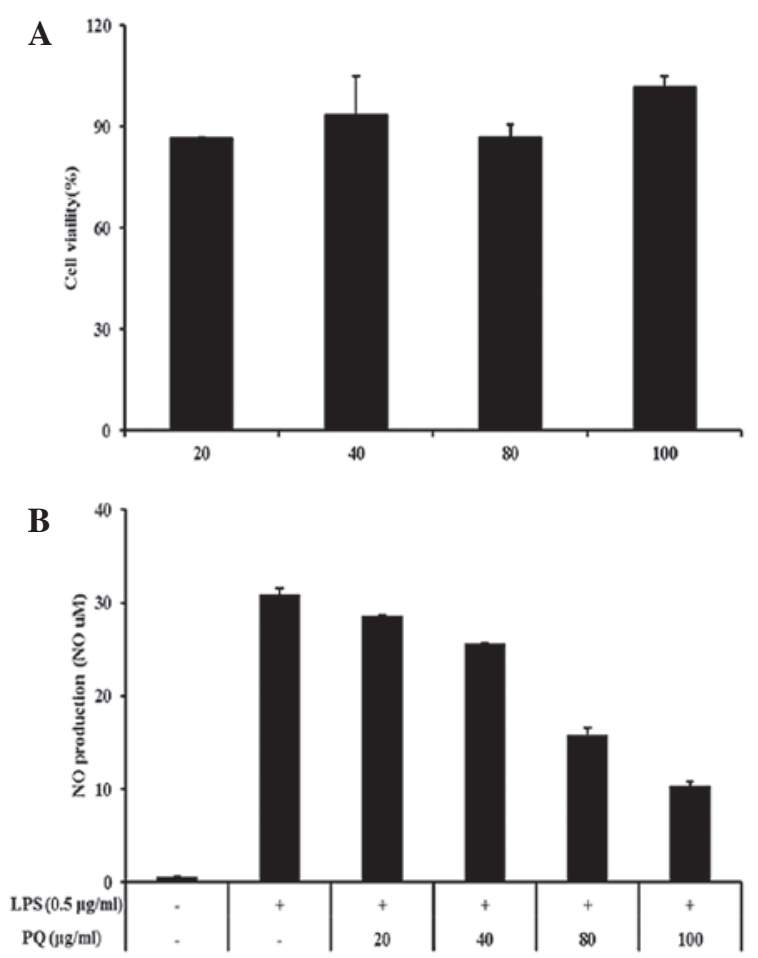

Figure 5. RAW 264.7 cells were treated with $20,40,80$ or $100 \mu \mathrm{g} / \mathrm{ml} P Q$ for $24 \mathrm{~h}$. (A) Survival rates were tested by MTT assay. (B) PQ inhibited NO production in the LPS-stimulated RAW 264.7 cells. The cells were induced by PQ $(20,40,80$ or $100 \mu \mathrm{g} / \mathrm{ml})$ for $1 \mathrm{~h}$ and treated with LPS $(0.5 \mu \mathrm{g} / \mathrm{ml})$, incubation for $24 \mathrm{~h}$. Values are expressed as the mean \pm standard deviation. ${ }^{\# \#} \mathrm{P}<0.01$, compared with $\mathrm{NC}$; ${ }^{*} \mathrm{P}<0.05$ and ${ }^{* *} \mathrm{P}<0.01$, compared with OVA. NO, nitric oxide; LPS, lipopolysaccharide; PQ, Picrasma quassioides; MTT, 3-(4,5-dimethylthiazol-2-yl)-2,5-diphenyltetrazolium bromide.

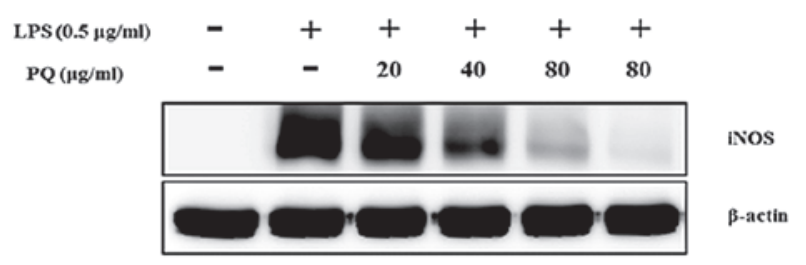

Figure 6. PQ reduced the levels of iNOS in the LPS-stimulated RAW264.7 cells. The expression levels of iNOS were assessed by western blot analysis. Detection of $\beta$-actin was performed to confirm the equal loading of proteins. LPS, lipopolysaccharide; PQ, Picrasma quassioides; iNOS, inducible nitric oxide synthase.

were almost undetectable in the non-stimulated cells. The LPS-stimulated cells exhibited increased iNOS protein expression levels compared with those of the non-stimulated cells. However, the cells pre-treated with PQ showed an inhibition of the iNOS expression in a concentration-dependent manner.

\section{Discussion}

The present study evaluated the effects of PQ on inflammatory responses using an OVA-sensitized/challenged allergic asthma murine model and LPS-stimulated RAW264.7 cells. Administration of PQ resulted in a significant reduction in the elevated inflammatory cell counts, AHR, and levels of Th2 cytokines and IgE induced by the OVA challenge. PQ attenuated the infiltration of inflammatory cells into the airway and mucus hypersecretion. In addition, the LPS-stimulated RAW264.7 cells exhibited significantly reduced levels of NO production when treated with PQ. Furthermore, PQ inhibited the overexpression of iNOS caused by LPS treatment.

Airway inflammation is an important feature of allergic asthma. In the initiation or development of airway inflammation, Th2 cytokines are central in causing the release of various inflammatory mediators, including IL-4, IL-5 and IL-13 (7-10). These cytokines are involved in the maturation and activation of eosinophils, B-cell isotype switching and the release of chemokines and chemoattractants, which result in the phenotypes of allergic asthma, including airway inflammation, AHR and airway remodeling (11-14). In the present study, PQ reduced the number of inflammatory cells, AHR and the levels of $\operatorname{IgE}$ as a result of the reduction in the levels of IL-4, IL-5 and IL-13. These finding indicate that PQ effectively suppressed airway inflammation via the downregulation of Th2 cytokines. These results were consistent with the histological analysis of the lung tissue in the present study. The lung tissue from the PQ-treated mice exhibited a reduction in the recruitment of inflammatory cells into the airway and mucus production in the airway compared with those in the OVA-sensitized/challenged mice.

NO, an important inflammatory mediator, is a gaseous molecule synthesized by NOS that has numerous detrimental functions. NOS has three isoforms, constitutive NOS, endothelial NOS and iNOS. Of the NOS isoforms, iNOS is closely associated with the production of NO (15). A previous study has demonstrated that overproduction of NO acts as a crucial mediator of airway inflammation in allergic asthma (16). NO contributes to the infiltration of inflammatory cells, including neutrophils, macrophages and eosinophils (8). In addition, NO is a potent stimulator of the Th2 responses of allergic asthma. According to a previous study, increased NO production induced by iNOS aggravated the allergic asthmatic responses, including airway inflammation and AHR, via the elevation of the levels of Th2 cytokine release. In a previous study, iNOS knockout mice exhibited a reduction in NO production, resulting in a decrease in the Th2 cytokine levels in an allergic asthma model (1). Therefore, numerous anti-inflammatory materials have been developed that focus on the suppression of iNOS expression. A number of natural products have been investigated for their anti-inflammatory effects using numerous experimental models (3-6). In the present study, PQ inhibited the elevated production of NO in LPS-stimulated RAW264.7 cells in a concentration-dependent manner. PQ also suppressed the overexpression of iNOS induced by LPS treatment. These results indicate that PQ effectively inhibits inflammatory responses via the suppression of iNOS expression.

Numerous oriental medicine practitioners have administered herbal treatments to patients to cure various diseases and PQ has been used as a traditional herbal remedy for several disorders, particularly inflammatory diseases (5). The present study provided evidence of the anti-inflammatory effects of PQ using an OVA-sensitized/challenged allergic asthma murine model and LPS-stimulated RAW264.7 cells. These effects were consistent with the results of a previous study (6). Fan et al (6) demonstrated that PQ possesses protective effects 
against carrageenan-induced paw edema, xylene-induced ear edema and adjuvant-induced arthritis.

In conclusion, PQ effectively suppressed airway inflammation induced by OVA challenge in the present study. The anti-inflammatory effects of PQ were considered to be associated with the suppression of iNOS expression based on the results of the in vitro experiments. Therefore, this study suggests that PQ may be a potent therapeutic agent for airway inflammatory diseases, including allergic asthma.

\section{Acknowledgements}

This study was supported by a grant from the Korea Research Institute of Bioscience and Biotechnology Research Initiative program (grant no. KGM1221312) of the Republic of Korea.

\section{References}

1. Prado CM, Martins MA and Tibério IF: Nitric oxide in asthma physiopathology. ISRN Allergy 2011: 832560, 2011.

2. Schuijs MJ, Willart MA, Hammad H and Lambrecht BN: Cytokine targets in airway inflammation. Curr Opin Pharmacol 13: 351-361, 2013.

3. Jiao WH, Gao H, Zhao F, Lin HW, Pan YM, Zhou GX and Yao XS: Anti-inflammatory alkaloids form the stems of Picrasma qussioides BENNET. Chem Pharm Bull (Tokyo) 59: 359-364, 2011.

4. Yin Y, Lee SK and Wang MH: Isolation and biological activities of an alkaloid compound (3-methylcanthin-5,6-dione) from Picrasma quassiodes (D.Don) Benn. Nat Prod Sci 17: 5-9, 2011.

5. Liu JF, Shao M, Zhai DW, Liu K and Wu LJ: Protective effect of 4-methoxy-5-hyfuoxycanthin-6-one, a natural alkaloid, on dextran sulfate sodium-induced rat colitis. Planta Med 75: 142-145, 2009.

6. Fan H, Qi D, Yang M, Fang H, Liu K and Zhao F: In vitro and in vivo anti-inflammatory effects of 4-methoxy-5-hydroxycanthin-6-one, natural alkaloid from Picrasma quassioides. Phytomedicine 20: 319-323, 2013.
7. Ra J,Lee S, Kim HJ, Jang YP, Ahn H and Kim J: Bambusae Caulis in Taeniam extract reduces ovalbumin-induced airway inflammation and T helper 2 responses in mice. J Ethnopharmacol 128: 241-247, 2010.

8. Nader MA, El-Awady MS, Shalaby AA and El-Aqamy DS: Sitagliptin exerts anti-inflammatory and anti-allergic effects in ovalbumin-induced murine model of allergic airway disease. Naunyn Schmidebergs Arch Pharmacol 385: 909-919, 2012.

9. Lee MY, Shin IS, Jeon WY, Lim HS, Kim JH and Ha H: Pinellia ternate Breitenbach attenuates ovalbumin-induced allergic airway inflammation and mucus secretion in a murine model of asthma. Immunopharmacol Immunotoxicol 35: 410-418, 2013.

10. Schmudde I, Laumonnier Y and Köhl J: Anaphylatoxins coordinate innate and adaptive immune responses in allergic asthma. Semin Immunol 25: 2-11, 2013.

11. Shin IS, Lee MY, Lim HS, Ha H, Seo CS, Kim JC and Shin HK: An extract of Crataegus pinnatifida fruit attenuates airway inflammation by modulation of matrix metalloproteinase-9 in ovalbumin induced asthma. PLoS One 7: e45734, 2012.

12. Lee YT, Lee SS, Sun HL, Lu KH, Ku MS, Sheu JN, Ko JL and Lue KH: Effect of the fungal immunomodulatory protein FIP-fve on airway inflammation and cytokine production in mouse asthma model. Cytokine 61: 237-244, 2013.

13. Simoes DC, Xanthou G, Petrochilou K, Panoutsakopoulou V, Roussos C and Gratziou C: Osteopontin deficiency protects against airway remodeling and hyperresponsiveness in chronic asthma. Am J Respir Crit Care Med 179: 894-902, 2009.

14. Shen JJ, Chiang MS, Kuo ML, Leu YL, Hwang TL, Liou CJ and Huang WC: Partially purified extract and viscolin from Viscum coloratum attenuate airway inflammation and eosinophil infiltration in ovalbumin-sensitized mice. J Ethnopharmacol 135: 646-653, 2011.

15. Koarai A, Ichinose M, Sugiura H, Tomaki M, Watanabe M, Yamagata S, Komaki Y, Shirato K and Hattori T: iNOS depletion completely diminishes reactive nitrogen-species formation after an allergic response. Eur Respir J 20: 609-616, 2002.

16. Deshane J, Zmijewski JW, Luther R, Gaggar A, Deshane R, Lai JF, Xu X, Spell M, Estell K, Weaver CT, Abraham E, Schwiebert LM and Chaplin DD: Free radical-producing myeloid-derived regulatory cells: potent activators and suppressors of lung inflammation and airway hyperresponsiveness. Mucosal Immunol 4: 503-518, 2011. 